\title{
Reversing factor Xa inhibitors - clinical utility of andexanet alfa
}

\author{
Scott Kaatz' \\ Hardik Bhansali' \\ Joseph Gibbs ${ }^{2}$ \\ Robert Lavender ${ }^{3}$ \\ Charles E Mahan ${ }^{4}$ \\ David G Paje ${ }^{5}$
}

'Division of Hospital Medicine, ${ }^{2}$ Department of Internal Medicine, Henry Ford Hospital, Detroit, MI, ${ }^{3}$ Division of General Internal Medicine, University of Arkansas for Medical Sciences, Little Rock, AR, ${ }^{4}$ University of New Mexico, Presbyterian Healthcare Services, Albuquerque, NM, ${ }^{5}$ Department of Internal Medicine, University of Michigan Medical School, Ann Arbor, MI, USA

Correspondence: Scott Kaatz Division of Hospital Medicine, Henry Ford Hospital, 2799 West Grand Boulevard, Detroit, MI 48202, USA $\mathrm{Tel}+\mathrm{I} 3139168144$

Fax + I 3139164460

Email skaatzl@hfhs.org
This article was published in the following Dove Press journal:

Journal of Blood Medicine

13 September 2017

Number of times this article has been viewed

\begin{abstract}
Approximately half of patients started on an oral anticoagulant in the USA now receive one of the newer direct oral anticoagulants (DOACs). Although there is an approved reversal agent for the direct thrombin inhibitor dabigatran, a specific reversal agent for the anti-factor Xa (FXa) DOACs has yet to be licensed. Unlike the strategy to reverse the only oral direct thrombin inhibitor with idarucizumab, which is a humanized monoclonal antibody fragment, a different approach is necessary to design a single agent that can reverse multiple anti-FXa medications. Andexanet alfa is a FXa decoy designed to reverse all anticoagulants that act through this part of the coagulation cascade including anti-FXa DOACs, such as apixaban, edoxaban and rivaroxaban, and indirect FXa inhibitors such as low-molecular-weight heparins. This narrative reviews the development of andexanet alfa and explores its basic science, pharmacokinetics/ pharmacodynamics, animal models, and human studies.
\end{abstract}

Keywords: factor Xa, DOAC, andexanet alfa, reversal, rivaroxaban, apixaban

\section{Introduction}

Direct oral anticoagulants (DOACs) are associated with lower rates of fatal bleeding and death from major bleeding compared to vitamin $\mathrm{K}$ antagonists (VKAs). ${ }^{1-3}$ All-cause mortality is also significantly reduced with DOACs, particularly among patients with nonvalvular atrial fibrillation. ${ }^{1,2}$ However, despite the preponderance of evidence of their safety, the absence of specific antidotes against DOACs has discouraged many patients and their physicians from embracing their emergence as long overdue alternatives to VKAs. ${ }^{4}$ Instead, the introduction of DOACs was quickly followed by an urgent need to develop agents that specifically reverse their anticoagulant effects.

This cautious approach is not surprising since oral anticoagulants have the highest rate of emergency room (ER) visits for adverse drug reactions with $\sim 17$ visits per 1,000 individuals per year with almost half resulting in hospitalization. ${ }^{5}$ Among elderly patients, a quarter to a third of all adverse drug reactions seen in the ER are related to anticoagulants. Although VKAs such as warfarin are implicated in a vast majority of these ER visits in older patients, with the increasing use of DOACs, rivaroxaban is now fifth and dabigatran tenth most common. ${ }^{5}$

Effective reversal strategies have been available for VKAs for decades, initially using vitamin $\mathrm{K}$ and fresh frozen plasma, and then later on with the addition of 4-factor prothrombin complex concentrate as the preferred agent for life-threatening bleeding. ${ }^{6}$ For the oral direct thrombin inhibitor, dabigatran, a humanized antibody fragment, idarucizumab, was recently developed as its specific antidote and is now 
approved for use. ${ }^{7}$ For oral direct factor Xa (FXa) inhibitors, including apixaban, edoxaban, and rivaroxaban, an agent that specifically targets these drugs is currently not yet available. For this reason, the US Food and Drug Administration (FDA) gave a breakthrough and fast track status to the development of andexanet alfa, a new investigational drug that binds to FXa inhibitors and reverses their anticoagulant effects. Since it binds compounds with a high affinity for FXa, it is also being evaluated as an antidote against indirect FXa inhibitors, such as low-molecular-weight heparin (LMWH), a parenteral anticoagulant that has been around for 2 decades and is only partially reversed by protamine sulfate.

\section{Structure, preclinical trials, pharmacokinetics, pharmacodynamics, and manufacturing}

Andexanet alfa is being developed by Portola Pharmaceuticals and is also known by the brand name Annexa. It is a rationally designed, bioengineered, recombinant modified protein intended to serve as a universal antidote against direct FXa inhibitors and against indirect FXa inhibitors. Scientifically, it has been referred to as PRT064445 or PRT4445, and in early medical literature as "r-Antidote". Andexanet is expressed in its mature functional form without the need for activation via the intrinsic or extrinsic coagulation pathways. ${ }^{8}$

\section{Structure and design}

To understand andexanet alfa's action, we briefly review the mechanism of action of FXa and FXa inhibitors. FXa adheres to the membrane surface along with cofactor Va and prothrombin in the presence of calcium to form the prothrombinase complex. This assembly of clotting factors increases the conversion of prothrombin to thrombin. FXa's N-terminal gamma-carboxyglutamic acid (Gla) domain enables it to adhere to surface membranes so that it can assemble with factor Va and prothrombin. FXa also has an active serine site that cleaves prothrombin to thrombin. The indirect FXa inhibitors, including fondaparinux and LMWH, conformationally change the structure of antithrombin III (ATIII), which makes this natural anticoagulant 1,000-fold more effective at blocking the serine active site of FXa and prevents conversion of prothrombin to thrombin. The direct FXa inhibitors, such as apixaban, betrixaban, edoxaban, and rivaroxaban, are small molecules. They directly and reversibly block the active serine site of FXa (Figure 1). ${ }^{9}$

Andexanet alfa is a recombinant modified FXa decoy molecule developed as a truncated form of enzymatically

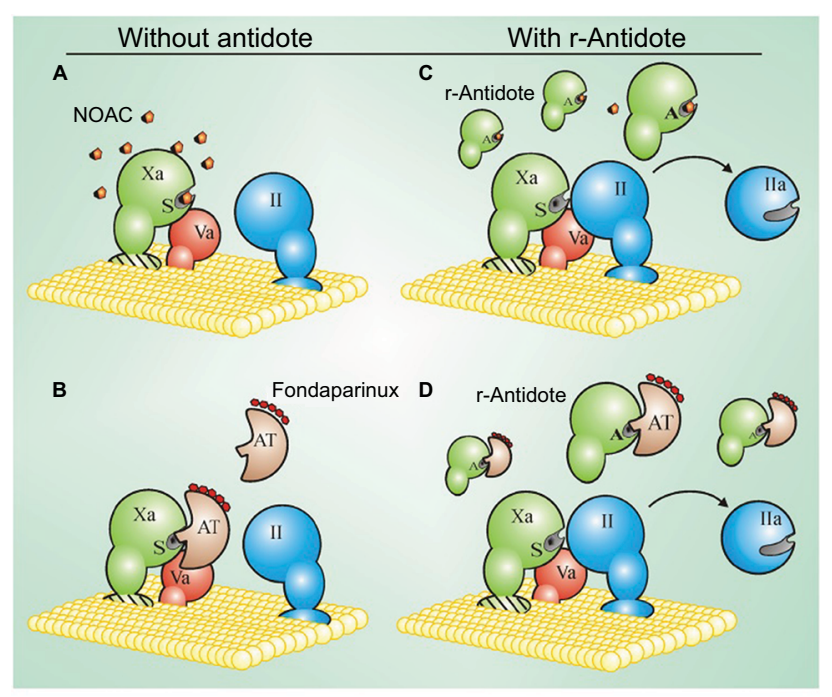

Figure I Mechanism of action of r-Antidote. ${ }^{9}$

Notes: (A) The prothrombinase complex, which consists of factor $\mathrm{Xa}$ and its cofactor factor $V a$, assembles on a membrane surface where it converts prothrombin (II) to thrombin (Ila). The membrane-binding Gla domain (hatched oval) and active site $S$ of factor $X a$ are essential for factor $X a$ function. Factor $X a-$ directed NOACs bind reversibly to the active site of factor $X a$ and attenuate its capacity to activate II. (B) Fondaparinux, a synthetic pentasaccharide, binds AT and catalyzes the inhibition of factor $\mathrm{Xa}$, thus attenuating prothrombin activation. (C) $r$-Antidote, which has its active $S$ converted to $A$ to prevent procoagulant activity, binds the NOACs but does not compete with factor $X a$ for incorporation into the prothrombinase complex because it lacks the membrane-binding Gla-domain. By binding NOACs, r-Antidote reverses factor $\mathrm{Xa}$ inhibition and restores that capacity of prothrombinase to generate thrombin and to effect hemostasis. (D) $r$-Antidote competes with factor $X a$ for fondaparinux-activated antithrombin, thereby allowing prothrombinase to generate thrombin. Reprinted with permission from Yeh $\mathrm{CH}$, Fredenburgh JC, Weitz Jl. The real decoy: an antidote for factor Xa-directed anticoagulants. Circ Res. 2013;1I3(8):954-957. Available from:http:// circres.ahajournals.org/content/1/3/8/954.long. Promotional and commercial use of the material in print, digital or mobile device format is prohibited without the permission from the publisher Wolters Kluwer. Please contact healthpermissions@ wolterskluwer.com for further information. ${ }^{9}$

Abbreviations: A, alanine; AT, antithrombin; NOACs, new oral anticoagulants; S, serine.

inactive FXa. The N-residue was retained to minimize the risk of immunogenicity. ${ }^{8}$ Compared to native FXa, structural modifications were undertaken, including removal of a 34-residue fragment containing Gla, which lowers the overall molecular weight of the compound and shortens the protein. This eliminates its potential to bind to membrane surfaces and assemble the prothrombinase complex, thus reducing its procoagulant potential. The absence of the Gla domain also prevents andexanet alfa from taking up space on phospholipid surface membranes, thereby allowing native FXa to bind and assemble the prothrominase complex. Finally, a mutation at the active binding site of andexanet from serine to alanine allows it to effectively bind FXa inhibitors, while at the same time eliminating its ability to cleave prothrombin and generate thrombin. Therefore, andexanet alfa cannot convert prothrombin to thrombin because it is unable to bind to phospholipid membrane surfaces and assemble with factor Va and prothrombin, and because it cannot convert prothrombin to thrombin because of alterations at the cleavage site (Figure 2). 


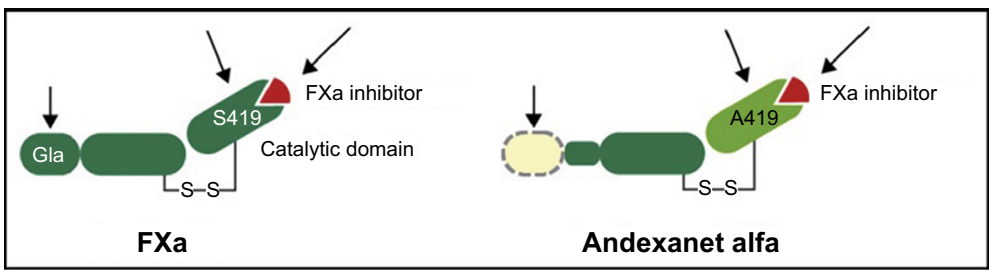

Figure 2 Design of andexanet alfa.

Notes: Serine, the active site of FXa, was substituted with alanine, rendering the molecule unable to cleave and activate prothrombin. The Gla domain of FXa was removed to prevent its assembly into the prothrombinase complex, thus removing any anticoagulant effects. Copyright @2016 Milling TJ Jr, Kaatz S. Preclinical and clinical data for factor Xa and "Universal" reversal agents. Am J Med. 20I6; I29(IIs):S80-S88. ${ }^{26}$

Abbreviations: $\mathrm{FXa}$, factor $\mathrm{Xa}$; Gla, gamma-carboxyglutamic acid.

\section{Preclinical trials}

In 2008, preclinical studies suggested that andexanet was effective in correcting coagulation parameters, such as prothrombin time (PT) and the international normalized ratio (INR) for apixaban, rivaroxaban, and betrixaban and the activated partial thromboplastin time (aPTT) for enoxaparin. ${ }^{10}$ Subsequent in vitro studies showed that andexanet effectively reversed enoxaparin and fondaparinux in human and rat plasma and in vivo rat models. ${ }^{11,12}$ In rat models given rivaroxaban, andexanet administration effectively reduced the free fraction of rivaroxaban. Use of andexanet in mouse tail models anticoagulated with aspirin and rivaroxaban also decreased anti-FXa activity and reduced blood loss by $>80 \%$ measured 15 minutes after its administration. In rabbit liver laceration models anticoagulated with rivaroxaban, andexanet reduced blood loss by $>85 \%$, corrected both free rivaroxaban levels, and reduced anti-FXa by $98 \%$, aPTT by $66 \%$, and PT levels by $74 \%$, while recombinant factor VIIa, at $150 \mu \mathrm{g} / \mathrm{kg}$, reduced PT by $85 \%$ and aPTT by $54 \%$ but did not reduce bleeding. ${ }^{13,14}$ Andexanet alone at the highest concentrations tested did not have any effect on PT or on a thrombin generation assay that tested FXa activity and the effect upon the prothrombinase complex. ${ }^{8}$ In 2015, andexanet also demonstrated the reversal of unfractionated heparin (UFH) in human plasma reversing anti-FXa and anti-factor IIa while ciraparantag (PER977) had no effect. ${ }^{15}$ As expected, protamine also reversed UFH; however, at higher doses, it also exhibited strong anticoagulant effects, which is an often overlooked adverse effect of protamine use in the clinical setting. ${ }^{15}$ In summary, andexanet corrected coagulation assays, restored hemostasis, and reduced blood loss in animal models.

\section{Pharmacology, pharmacokinetics, and pharmacodynamics}

Andexanet is given via the intravenous (IV) route with either a $400 \mathrm{mg}$ initial bolus and a $480 \mathrm{mg}$ infusion over 2 hours
( $4 \mathrm{mg} /$ minute) or an $800 \mathrm{mg}$ initial bolus and a $960 \mathrm{mg}$ infusion over 2 hours ( $8 \mathrm{mg} /$ minute). The drug needs refrigeration for storage. Onset is rapid between 2 and 5 minutes. Elimination half-life appears to be between 30 and 60 minutes. Little is known on the elimination (ie, metabolism and excretion) of the drug at this point.

Andexanet has high affinity for the FXa inhibitors and competes with native FXa to bind the anticoagulants, which frees uninhibited FXa to assemble into the prothrombinase complex and generate thrombin. Andexanet has a short halflife and requires a bolus followed by an infusion to allow adequate time to achieve a hemostatic plug (Figure 3). ${ }^{16,17}$ The Xa inhibitor DOAC serum level rises within a couple of hours after administration of andexanet and the drug is not "cleared" with the reversal agent, unlike what an antibody such as idarucizumab does with dabigatran. ${ }^{17,18}$

Initially, upon bolus administration, andexanet appears to elevate endogenous thrombin potential, although the mechanism is not yet known. However, the compound itself does not appear to demonstrate any anticoagulant or procoagulant properties. The transient increases in prothrombin fragments 1 and 2, D-dimer, and thrombin-antithrombin levels may be the result of andexanet binding to tissue factor pathway inhibitor (TFPI), which is an endogenous inhibitor of FXa.$^{19}$ Typically, these transient elevations return to normal within 1-3 days. ${ }^{17}$ In theory, andexanet may have potential interactions, such as with circulating ATIII not in the presence of heparins, factor $\mathrm{V}$, protein $\mathrm{S}$, protein $\mathrm{Z}$ inhibitor, and TFPI, but the significance of these interactions requires further study. ${ }^{8}$

It does not appear that andexanet elicits antibody formation against factor $\mathrm{X}$ or FXa. While neutralizing antibodies against andexanet have not been detected, non-neutralizing antibodies have been detected and typically appear within 30 days of andexanet administration. However, antibody titers are low suggesting that andexanet has little immunogenicity. ${ }^{16,17}$ Patient studies with long-term follow-up, in which andexanet is repeatedly administered, are needed to determine if the 


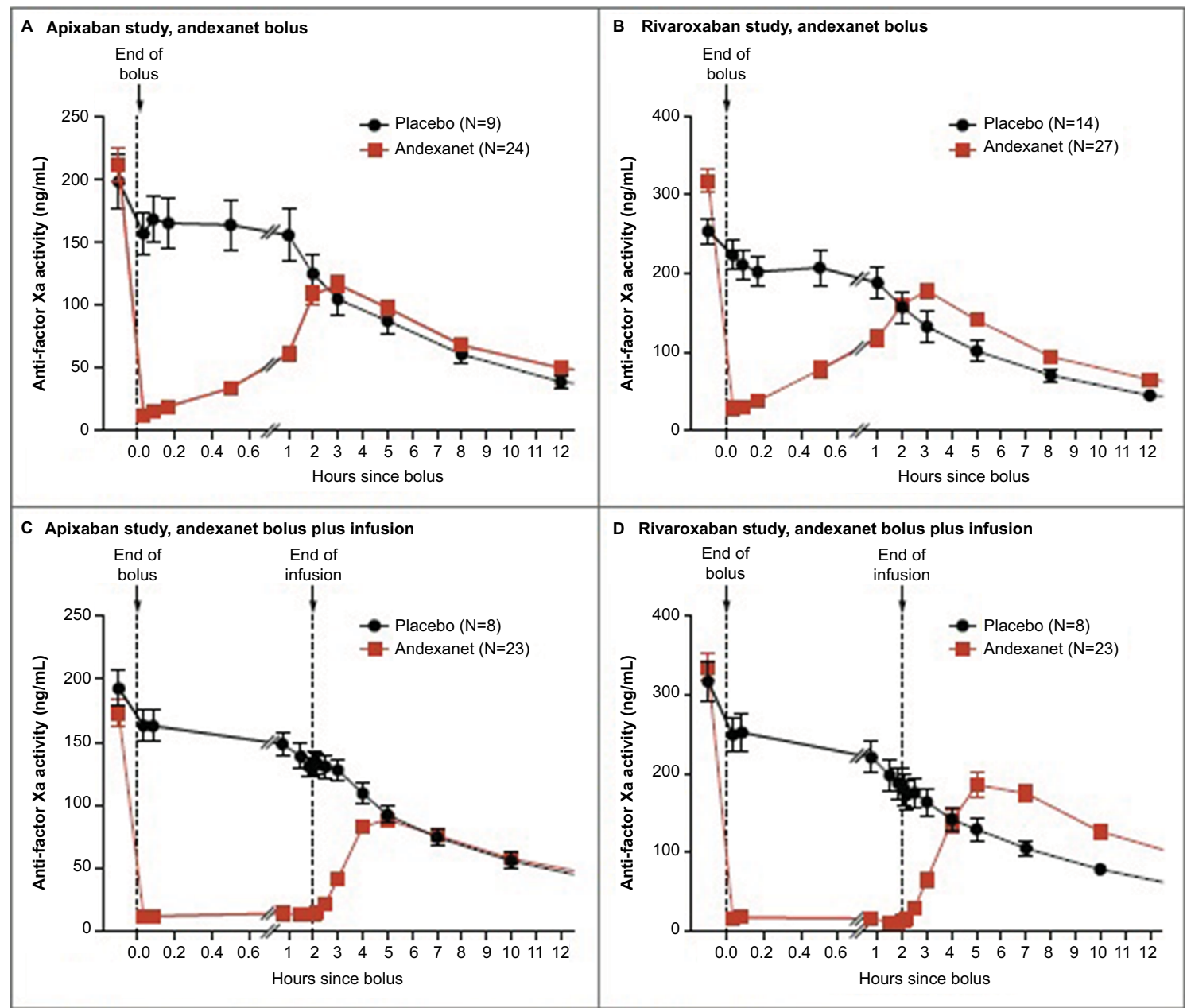

Figure 3 Time courses of anti-factor Xa activity before and after administration of andexanet. ${ }^{17}$

Notes: Anti-factor Xa activity among persons who had received anticoagulation treatment with apixaban or rivaroxaban was measured before and after the administration of andexanet or placebo on study day 4. Dashed lines indicate the end of administration of the bolus or infusion. (A) Data from participants in the apixaban study (ANNEXA-A) who received andexanet, as a $400 \mathrm{mg}$ intravenous bolus, or placebo. (B) Data from participants in the rivaroxaban study (ANNEXA-R) who received andexanet, as an $800 \mathrm{mg}$ intravenous bolus, or placebo. (C) Data from participants in the apixaban study who received andexanet, as a $400 \mathrm{mg}$ intravenous bolus with a $4 \mathrm{mg} / \mathrm{minute}$ infusion for 120 minutes, or placebo. (D) Data from participants in the rivaroxaban study who received andexanet, as an $800 \mathrm{mg}$ intravenous bolus with an $8 \mathrm{mg} / \mathrm{minute}$ infusion for 120 minutes, or placebo. Different scales along the $x$-axis in each graph are used to enable visualization of the immediate, short-term dynamics as well as the longer-term dynamics of anti-factor $\mathrm{Xa}$ activity after andexanet treatment; the points on the graph represent the mean anti-factor $\mathrm{Xa}$ activity level, and I bars indicate the standard error. There was a significant difference $(P<0.05)$ in the percent change in anti-factor Xa activity (relative to the pre-bolus activity level) between andexanet and placebo until 2 hours after administration of the bolus or infusion. From N Engl J Med., Andexanet alfa for the reversal of factor Xa inhibitor activity.20I5;373(25):24I3-2424. Copyright @ 20I5 Massachusetts Medical Society. Reprinted with permission from Massachusetts Medical Society. ${ }^{17}$

generation of cross-reactive antibodies to native factor X, FXa, or andexanet occurs.

\section{Manufacturing concerns}

Andexanet is produced from Chinese hamster ovary $(\mathrm{CHO})$ cells, and Portola Pharmaceuticals is collaborating with the manufacturers CMC Biologics (Copenhagen, Denmark) and Lonza (Visp, Switzerland) to provide the recombinant protein. One of the FDA's concerns from their first review was the availability of drug once approved, with estimates that only $10 \%$ of US hospitals could be supplied. Portola is working with its manufacturing partners to increase production to meet anticipated global needs. As of May 2017, andexanet produced from a second manufacturing site, which uses a second generation manufacturing process, is undergoing validation and will likely be used in the Annexa-4 study (Charles E Mahan, personal communication, from Portola Pharmaceuticals 5-25-17).

\section{Early-phase studies Phase I}

In a dose-ranging study, 32 healthy volunteers were randomized to receive a single IV bolus dose of placebo or andexanet alfa at doses of $30,90,300$, or $600 \mathrm{mg} .{ }^{20}$ Blood samples from these volunteers were then obtained, mixed with $50 \mathrm{ng} / \mathrm{mL}$ of rivaroxaban, and then assessed for anti-FXa activity. Andexanet alfa reversed anti-FXa activity and restored thrombin generation in a dose-dependent manner. No thrombotic 
events or death occurred. The only serious adverse event was pneumonia, which developed in one subject $\sim 3$ weeks after receiving andexanet. There were three nonserious infusion site reactions without anaphylaxis, which occurred in two subjects who received $90 \mathrm{mg}$ of andexanet and in one subject randomized to placebo.

\section{Phase II}

During a series of Phase II clinical trials, healthy adults from ages 18 to 45 years were enrolled in randomized, doubleblind, placebo-controlled experiments to assess andexanet's safety and efficacy. ${ }^{19,21-23}$ On days $1-6$, the subjects were initially treated to achieve steady state with an FXa inhibitor, which could be apixaban, rivaroxaban, betrixaban, edoxaban, or enoxaparin. Then, on day 6 , they received one of the six different dose regimens of andexanet alfa or matched placebo. The dose regimens were either IV bolus only or IV bolus followed by a continuous infusion.

Across these trials, andexanet alfa was well tolerated. No thrombotic, serious, or severe adverse events occurred. Most adverse events were mild-to-moderate infusion reactions that generally did not require intervention or dose adjustment. With regard to immunogenic effect, there were no antibodies detected against endogenous human factor X or FXa. However, $\sim 12 \%$ of subjects had low-titer, nonneutralizing antibodies to andexanet alfa. ${ }^{23}$ Within 2 minutes of administration, andexanet alfa demonstrated a dose-dependent reversal of the anticoagulant effects of all FXa inhibitors, including reduction in anti-FXa activity and restoration of thrombin generation and clotting times. Also, following the initial IV bolus with continuous infusion of andexanet resulted in a sustained reduction of anti-FXa activity, which returned to placebo levels $\sim 2$ hours after stopping the infusion.

The reduction in anti-FXa activity with andexanet directly correlated with the decline in plasma concentrations of unbound FXa inhibitor. Subjects who were on apixaban $5 \mathrm{mg}$ twice daily for 6 days had a $65 \%$ decline in anti-FXa activity within 2 minutes of receiving $90 \mathrm{mg}$ of andexanet, which bound $66 \%$ of apixaban in plasma. ${ }^{19}$ Similarly, those who were on rivaroxaban $20 \mathrm{mg}$ daily for 6 days had a $53 \%$ decline in anti-FXa activity following receipt of $420 \mathrm{mg}$ of andexanet, which reduced the plasma concentration of unbound rivaroxaban by $51 \% .{ }^{24}$ These results were the basis for establishing the 1:1 stoichiometric ratio necessary for andexanet to reverse the effect of FXa inhibitors. The optimal dose of andexanet required against each $\mathrm{FXa}$ inhibitor varied and was primarily determined by each drug's steady state plasma concentration and volume of distribution. Therefore, in subsequent Phase II trials, the dose of andexanet selected for participants on apixaban $5 \mathrm{mg}$ twice daily was $400 \mathrm{mg}$ bolus followed by a continuous infusion of $4 \mathrm{mg} / \mathrm{minute}$ for 2 hours, while those who were on rivaroxaban $20 \mathrm{mg}$ daily were given an $800 \mathrm{mg}$ bolus followed by a continuous infusion of $8 \mathrm{mg} / \mathrm{minute}$ for 2 hours. ${ }^{17,19}$

\section{Phase III}

\section{ANNEXA-A and ANNEXA-R trials}

Two trials were conducted to investigate the efficacy and safety of andexanet alfa to reverse the anticoagulation effects of apixaban and rivaroxaban in healthy older volunteers. The ANNEXA trials (andexanet alfa, a novel antidote to the anticoagulation effects of FXa inhibitors) were divided into the ANNEXA-A arm for apixaban and ANNEXA-R arm for rivaroxaban. ${ }^{17}$ Both were randomized, double blind, and placebo controlled. A total of 145 healthy volunteers between the ages of 50 and 75 years were randomly assigned (3:1 ratio for apixaban arm and 2:1 ratio for rivaroxaban arm) to receive either andexanet or placebo. Each arm had two parts in which participants received IV andexanet bolus only in part 1 and a continuous 120 minute infusion of andexanet after the bolus in part 2. The doses of andexanet were selected based on Phase II trials, which determined that the dose of andexanet to reverse effects of rivaroxaban is higher compared to apixaban due to the higher initial concentration and larger volume of distribution of rivaroxaban.

Participants received apixaban $5 \mathrm{mg}$ orally twice a day in ANNEXA-A arm for 3.5 days to reach steady-state plasma levels. Andexanet bolus of $400 \mathrm{mg}$ was administered intravenously 3 hours after the last dose of apixaban on day 4 in part 1 . The bolus was followed by continuous infusion of andexanet at $4 \mathrm{mg} /$ minute for 120 minutes in part 2. Anti-FXa activity was significantly reduced after andexanet bolus compared to after placebo (mean $[ \pm \mathrm{SD}]$ reduction, $94 \pm 2$ vs $21 \pm 9 \% ; P<0.001)$. The reversal of antiFXa activity lasted for 2 hours after administration of the andexanet bolus and gradually returned to levels seen in the placebo group. Andexanet infusion after bolus resulted in greater reduction of anti-FXa activity than placebo $(92 \pm 3$ vs $33 \pm 6 \%, P<0.001)$. Anti-FXa activity decreased in the placebo group at the expected clearance rate of apixaban. Reversal of anti-FXa activity persisted for 1-2 hours after andexanet bolus and then returned to placebo levels (Figure 3). All the participants with andexanet had at least $80 \%$ reversal of anti-FXa activity. None of participants with placebo had an $\geq 80 \%$ decrease of anti-FXa activity. Andexanet also significantly restored thrombin generation compared to 
placebo $(1,323.2 \pm 335.4$ vs $88.2 \pm 125.8 \mathrm{nM}$ minute, $P<0.001)$. Thrombin generation increased to levels in the normal range in $100 \%$ of participants with andexanet and in $11 \%$ of participants with placebo. Thrombin generation was significantly higher in participants who received bolus plus infusion of andexanet than in participants with placebo $(1,193.1 \pm 263.3$ vs $189.4 \pm 184.8 \mathrm{nM}$ minute, $P<0.001)$. Thrombin generation increased to levels in the normal range in $100 \%$ of participants with andexanet bolus plus infusion, compared to $25 \%$ of participants with placebo. Unbound apixaban was reduced significantly after andexanet bolus compared to placebo (9.3 vs $1.9 \mathrm{ng} / \mathrm{mL}, P<0.001)$. Andexanet bolus plus infusion also reduced unbound apixaban significantly compared with placebo (6.5 vs $3.0 \mathrm{ng} / \mathrm{mL}, P<0.001)$. Unbound apixaban increased to placebo levels within 1-3 hours after andexanet bolus or infusion.

Participants received rivaroxaban $20 \mathrm{mg}$ orally once a day in ANNEXA-R arm for 4 days to reach steady-state plasma levels. Andexanet bolus of $800 \mathrm{mg}$ was administered intravenously 4 hours after the last dose of rivaroxaban on day 4 in part 1 . The bolus was followed by continuous infusion of andexanet at $8 \mathrm{mg} / \mathrm{minute}$ for 120 minutes in part 2. Anti-FXa activity reduced significantly after andexanet bolus than after placebo (mean $[ \pm \mathrm{SD}]$ reduction, $92 \pm 11 \mathrm{vs}$ $18 \pm 15 \% ; P<0.001)$. The reversal of anti-FXa activity lasted for 2 hours after administration of the andexanet bolus and gradually returned to levels seen in the placebo group. Andexanet infusion after bolus resulted in greater reduction of anti-FXa activity than placebo ( $97 \pm 2$ vs $45 \pm 12 \%$, $P<0.001)$. Anti-FXa activity decreased in the placebo group at the expected clearance rate of rivaroxaban. Reversal of antiFXa activity persisted for 1-2 hours after andexanet bolus and then returned to placebo levels (Figure 3). Andexanet also significantly restored thrombin generation compared to placebo $(1,314.2 \pm 331.2$ vs $173.9 \pm 104.2 \mathrm{nM}$ minute, $P<0.001)$. Thrombin generation increased to levels in the normal range in $96 \%$ of participants with andexanet and in $7 \%$ of participants with placebo. Thrombin generation was significantly higher in participants who received bolus plus infusion of andexanet compared with placebo (1,510.4 \pm 344.8 vs $264.4 \pm 140.7 \mathrm{nM}$ minute, $P<0.001)$. Thrombin generation increased to levels in the normal range in $100 \%$ of participants with andexanet bolus plus infusion and in $0 \%$ of participants with placebo. Unbound rivaroxaban was significantly reduced after andexanet bolus compared with placebo (23.4 vs $4.2 \mathrm{ng} / \mathrm{mL}, P<0.001)$. Andexanet bolus plus infusion also reduced unbound rivaroxaban significantly compared to placebo (30.3 vs $12.1 \mathrm{ng} / \mathrm{mL}, P<0.001)$. Unbound rivaroxaban increased to placebo levels within 1-3 hours after andexanet bolus or infusion (Table 1).

Participants were monitored for safety outcomes of thrombosis and bleeding for 6 weeks. There were no thrombotic events. Antibodies to FXa were not detected in any participants. Neutralizing antibodies to andexanet were not detected. Nonneutralizing antibodies to andexanet were seen in one of 44 placebo recipients and 17 of 101 andexanet recipients. These antibodies disappeared 15-30 days after andexanet, suggesting that andexanet has no significant immunogenicity. D-dimer and prothrombin fragments were transiently elevated but returned to normal range in 24-72 hours.

The ANNEXA-A and ANNEXA-R studies showed that andexanet rapidly restores $\mathrm{FXa}$ activity and thrombin generation in participants treated with apixaban and rivaroxaban, without serious side effects or clinical thrombosis.

\section{ANNEXA-4 study}

ANNEXA-4 (andexanet alfa, a novel antidote to the anticoagulation effects of FXa inhibitors) is an ongoing multicenter,

Table I Results from ANNEXA-A and ANNEXA-R trials ${ }^{17}$

\begin{tabular}{|c|c|c|c|c|c|c|c|c|}
\hline \multirow[t]{3}{*}{$\begin{array}{l}\text { Measures of reversibility of } \\
\text { apixaban and rivaroxaban }\end{array}$} & \multicolumn{4}{|c|}{$\begin{array}{l}\text { Andexanet after apixaban } \\
\text { administration (ANNEXA-A) }\end{array}$} & \multicolumn{4}{|c|}{$\begin{array}{l}\text { Andexanet after rivaroxaban } \\
\text { administration (ANNEXA-R) }\end{array}$} \\
\hline & \multicolumn{2}{|c|}{$\begin{array}{l}\text { ANNEXA-A } \\
\text { bolus arm }\end{array}$} & \multicolumn{2}{|c|}{$\begin{array}{l}\text { ANNEXA-A bolus with } \\
\text { infusion arm }\end{array}$} & \multicolumn{2}{|c|}{$\begin{array}{l}\text { ANNEXA-R } \\
\text { bolus arm }\end{array}$} & \multicolumn{2}{|c|}{$\begin{array}{l}\text { ANNEXA-R bolus with } \\
\text { infusion arm }\end{array}$} \\
\hline & Bolus & Placebo & $\begin{array}{l}\text { Bolus with } \\
\text { infusion }\end{array}$ & Placebo & Bolus & Placebo & $\begin{array}{l}\text { Bolus with } \\
\text { infusion }\end{array}$ & Placebo \\
\hline $\begin{array}{l}\text { Mean \% reduction in anti-factor } \\
\text { Xa activity }\end{array}$ & 94 & 21 & 92 & 33 & 92 & 18 & 97 & 45 \\
\hline $\begin{array}{l}\text { Mean thrombin generation } \\
\text { (nM minute) }\end{array}$ & I,323.2 & 88.2 & I,193.I & 189.4 & $1,3 \mid 4.2$ & 173.9 & $\mathrm{I}, 5 \mathrm{I} 0.4$ & 264.4 \\
\hline $\begin{array}{l}\text { Mean reduction in unbound drug } \\
\text { level }(\mathrm{ng} / \mathrm{mL})\end{array}$ & 9.3 & 1.9 & 6.5 & 3 & 23.4 & 4.2 & 30.3 & 12.1 \\
\hline
\end{tabular}

Note: From N Engl J Med., Andexanet alfa for the reversal of factor Xa inhibitor activity. 2015;373(25):2413-2424. Copyright @ 2015 Massachusetts Medical Society. Reprinted with permission from Massachusetts Medical Society. ${ }^{17}$ 
prospective, open-label, and single-group study in patients with acute life-threatening bleeding while taking an FXa inhibitor. ${ }^{16}$ Patients who need reversal for an urgent procedure or surgery are not evaluated in this study. Patients are eligible if they are at least 18 years old and have received any of the four FXa inhibitors, such as apixaban, rivaroxaban, edoxaban, and enoxaparin, in the last 18 hours before presentation. Acute major bleeding includes acute life-threatening bleeding with signs or symptoms of hemodynamic instability, acute bleeding with drop in hemoglobin of at least $2 \mathrm{~g} / \mathrm{dL}$ or hemoglobin level $<8 \mathrm{~g} / \mathrm{dL}$ if no baseline hemoglobin level was available, or acute bleeding in a critical organ or area. Patients are excluded if surgery is scheduled in $<12$ hours, intracranial hemorrhage in a patient with Glasgow coma score $<7$, intracranial hematoma of $\geq 60 \mathrm{~mL}$, major thrombotic event 2 weeks before enrollment, or patients who have received dabigatran, VKA, prothrombin complex concentrate, or whole plasma in the 7 days before enrollment. A total of 67 patients were reported in an interim analysis of whom 32 patients received rivaroxaban (median daily dose, $20 \mathrm{mg}$ ), 31 patients received apixaban (median daily dose, $5 \mathrm{mg}$ ), and four patients received enoxaparin. The mean age was 77 years, and all had history of thrombotic events and cardiovascular disease. Gastrointestinal was the primary bleeding site in 33 patients ( $49 \%$ ), intracranial in 28 patients $(42 \%)$, and other sites in six patients $(9 \%)$.

All eligible patients were treated with andexanet before anti-FXa levels were known. All patients who received andexanet were included in the safety analysis, and patients with baseline anti-FXa activity of $\geq 75 \mathrm{ng} / \mathrm{mL}$ (or $0.5 \mathrm{IU} / \mathrm{mL}$ for patients with enoxaparin) were included in the efficacy analysis (47 of 67 patients). The primary outcomes were percent change in the anti-FXa activity and rate of excellent or good hemostatic efficacy 12 hours after the andexanet infusion. Patients received an initial bolus followed by 2-hour infusion of andexanet. Bolus dose of $400 \mathrm{mg}$ and total infusion of $480 \mathrm{mg}$ were given if patients received apixaban or rivaroxaban $>7$ hours before andexanet. Dose was increased to bolus of $800 \mathrm{mg}$ and total infusion of $960 \mathrm{mg}$, if patients received enoxaparin, edoxaban, or rivaroxaban $<7$ hours before andexanet. Investigators were encouraged to resume anticoagulation if clinically indicated. Patient assessments were done before the bolus administration (baseline), at the end of bolus, at the end of the 2-hour infusion and at 4, 8 , and 12 hours after infusion was completed, as well as at 3 and 30 days. Anti-FXa activity and the plasma unbound fraction of FXa inhibitor were measured in this 12-hour period. Thrombin generation was measured at baseline, at 8 hours, at 12 hours, and on day 3. Patients with intracranial hemorrhage were followed with computed tomography or magnetic resonance imaging of the head, at baseline, 1 hours, 12 hours, and 30 days after the end of infusion. All adverse events, including thrombotic events and deaths, were collected during the 30-day study period.

Hemostatic efficacy was assessed based on the type of bleeding. For intracranial hemorrhage, $<20 \%$ increase in volume at 12 hours was considered excellent hemostasis and $20-35 \%$ increase in volume at 12 hours was considered good hemostasis. Subarachnoid and subdural bleeding was assessed similarly but using maximal hematoma thickness. For gastrointestinal bleeding, $<10 \%$ decrease in hemoglobin and hematocrit at 12 hours was considered excellent hemostasis and 10-20\% decrease in hemoglobin and hematocrit at 12 hours was considered good hemostasis. For visible bleeding and for musculoskeletal bleeding, cessation of bleeding within 1 hour after infusion was considered excellent hemostasis and cessation of bleeding within 4 hours after infusion was considered good hemostasis.

In the 26 patients who received rivaroxaban, the median anti-FXa activity decreased from $277.0 \mathrm{ng} / \mathrm{mL}$ at baseline to $16.8 \mathrm{ng} / \mathrm{mL}$ at the end of andexanet bolus (relative decrease [RD], 89\%; 95\% CI, 58-94), $30.6 \mathrm{ng} / \mathrm{mL}$ at the end of andexanet infusion (RD, 86\%; 95\% CI, 55-93), and $177.7 \mathrm{ng} / \mathrm{mL}$ at 4 hours after infusion (RD, 39\%; 95\% CI, 27-45). These results highlight the mechanism of andexanet and demonstrate the reversal of $\mathrm{Xa}$ inhibition during administration, which quickly dissipates once stopped. How this short-term reversal affects clinical hemostasis is encouraging with the interim analysis of the ANNEXA-4 study, but further results are needed. In the 20 patients who received apixaban, the median anti-FXa activity decreased from $149.7 \mathrm{ng} /$ $\mathrm{mL}$ at baseline to $10.3 \mathrm{ng} / \mathrm{mL}$ at the end of andexanet bolus (RD, 93\%; 95\% CI, 87-94\%) and to $12.5 \mathrm{ng} / \mathrm{mL}$ at the end of andexanet infusion (RD, 92; 95\% CI, 85-94). Anti-FXa activity recovered to $103.0 \mathrm{ng} / \mathrm{mL}$ at 4 hours after infusion (RD, 30\%; 95\% CI, 23-46) and remained at similar levels at 8 and 12 hours. In the single patient who received enoxaparin, the median anti-FXa activity decreased from $0.61 \mathrm{IU} / \mathrm{mL}$ at baseline to $0.15 \mathrm{IU} / \mathrm{mL}$ at the end of andexanet bolus, $0.19 \mathrm{IU} / \mathrm{mL}$ at the end of andexanet infusion, and $0.46 \mathrm{IU} / \mathrm{mL}$ at 4 hours after infusion.

Of the 47 patients included in the efficacy analysis, 37 patients had excellent or good hemostasis (79\%; 95\% CI, 64-89), with 31 patients achieving excellent hemostasis and six patients achieving good hemostasis 12 hours after the andexanet infusion. Nine patients had poor or no hemostatic 
efficacy, out of which five patients received rivaroxaban and four patients received apixaban. Of these patients, three patients had gastrointestinal bleeding, four patients had intracranial bleeding, and two patients had bleeding at other sites. Excellent or good hemostasis was achieved in $84 \%$ (95\% CI, 64-96) of cases with gastrointestinal bleeding and in $80 \%$ (95\% CI, 56-94) of cases with intracranial bleeding. Excellent or good hemostasis was achieved in $81 \%$ (95\% CI, 61-93) of patients who received rivaroxaban and in 75\% (95\% CI, 51-91) of patients who received apixaban. Out of five patients with the highest anti-FXa activity after andexanet infusion, four patients had received rivaroxaban and one patient had received apixaban. All these patients achieved excellent or good hemostasis, in spite of receiving the lower dose regimen of andexanet. The median anti-FXa activity decreased from $487.1 \mathrm{ng} / \mathrm{mL}$ at baseline to $327.4 \mathrm{ng} /$ $\mathrm{mL}$ after andexanet infusion.

Among all 67 patients who received andexanet, no one experienced an infusion reaction or developed antibodies to $\mathrm{FXa}$ or $\mathrm{X}$, or neutralizing antibodies to andexanet. In the 12 patients with thrombotic events, there was one patient with myocardial infarction, five patients with strokes, seven patients with deep venous thrombosis, and one patient with pulmonary embolism. Four of these events occurred within 3 days after andexanet infusion, whereas the rest occurred between 4 and 30 days. A total of 10 deaths (15\%) occurred, six were cardiovascular in nature and the other four patients were noncardiovascular. Anticoagulation was restarted in 18 patients $(27 \%)$ within 30 days, but among the 12 patients with a thrombotic event, only one patient started therapeutic anticoagulation before the event. It is likely that the lack of therapeutic anticoagulation rather than a rebound phenomenon was responsible for the thrombosis. However, some hypothesize that andexanet may block TFPI, which could lead to thrombosis. ${ }^{25}$

The ANNEXA-4 study showed that andexanet bolus and infusion result in rapid and significant reversal of anti-FXa activity in patients presenting with acute major bleeding while on an FXa inhibitor. Excellent or good hemostatic efficacy was achieved in $79 \%$ of patients and was maintained across various subgroups. Clinical hemostatic efficacy was sustained hours after andexanet infusion, which suggests that prolonged reversal of anti-FXa activity is not necessary for good hemostatic effects. There were no serious side effects associated with the administration of andexanet; however, thrombotic events and deaths were reported. A controlled trial is needed to assess whether rates of these events are higher than expected for a population that has inherently high risk for thrombotic events. ANNEXA-4 is an ongoing study, and further analysis after full enrollment may elucidate the relationship between reduction in anti-FXa activity and hemostatic efficacy.

\section{Summary}

Prescribing of DOACs that inhibit FXa is rapidly increasing over recent years. Available trial data suggest that even without a readily available reversal agent, DOACs are associated with lower major and fatal bleeding rates. However, because of growing utilization, the incidence of DOAC-associated bleeding has been increasing and is expected to continue to rise, therefore, warranting the need for a readily available reversal agent. Andexanet alfa is a rationally designed, bioengineered FXa decoy. It acts as a competitive inhibitor that binds both direct and indirect FXa inhibitors, thereby restoring the ability of native FXa to assemble on phospholipid membranes and form the prothrombinase complex. While there were initial concerns regarding potential prothrombotic properties and immunogenicity, results of early clinical studies on andexanet have been reassuring. A mutation of the active-site binding pocket from serine to alanine eliminates the ability for andexanet to cleave prothrombin to thrombin, while maintaining its ability to actively bind anticoagulant drugs that inhibit FXa. A minority of patients may develop low-titer non-neutralizing antibodies to andexanet alfa, but these are short lived and become undetectable within 15-30 days. Andexanet alfa reduces anti-FXa activity by at least $80 \%$ and achieves excellent or good hemostasis in most patients with life-threatening bleeding. The ANNEXA-4 study is an ongoing trial that will provide further insights regarding andexanet's clinical efficacy as well as the associated incidence of thrombotic and other adverse events.

Andexanet alfa is being developed as a universal antidote for both direct (apixaban, edoxaban, and rivaroxaban) and indirect (fondaparinux and LMWH) FXa inhibitors. While further data are still necessary to assess adverse outcomes in at-risk populations, available results from recent trials have been encouraging. Andexanet alfa has the potential to serve as a safe and an effective antidote for DOAC-related bleeding.

\section{Disclosure}

SK received a speaker's honorarium from Janssen, BoehringerIngelheim, Bristol Myer Squibb, Pfizer, CSL Behring, and Daiichi Sankyo; is a consultant in Boehringer Ingelheim, Bristol Myer Squibb, Pfizer, Janssen, Daiichi Sankyo, Portola, and Roche; and holds the board membership (nonprofit) in the Thrombosis and Hemostasis Societies of North America, 
AC Forum, National Certification Board of Anticoagulation Providers, and National Blood Clot Alliance Medical and Scientific Advisory Board. CEM received a speaker's honorarium from Janssen, Boehringer-Ingelheim, Bristol Myer Squibb, Pfizer, and Portola and is a consultant in Janssen, BoehringerIngelheim, Bristol Myer Squibb, Pfizer, and Portola. The other authors report no other conflicts of interest in this work.

\section{References}

1. Chai-Adisaksopha C, Hillis C, Isayama T, Lim W, Iorio A, Crowther M. Mortality outcomes in patients receiving direct oral anticoagulants: a systematic review and meta-analysis of randomized controlled trials. J Thromb Haemost. 2015;13(11):2012-2020.

2. Ruff CT, Giugliano RP, Braunwald E, et al. Comparison of the efficacy and safety of new oral anticoagulants with warfarin in patients with atrial fibrillation: a meta-analysis of randomised trials. Lancet. 2014;383(9921):955-962.

3. Caldeira D, Rodrigues FB, Barra M, et al. Non-vitamin K antagonist oral anticoagulants and major bleeding-related fatality in patients with atrial fibrillation and venous thromboembolism: a systematic review and meta-analysis. Heart. 2015;101(15):1204-1211.

4. Hunt BJ, Levi M. Engineering reversal - finding an antidote for direct oral anticoagulants. N Engl J Med. 2016;375(12):1185-1186.

5. Shehab N, Lovegrove MC, Geller AI, Rose KO, Weidle NJ, Budnitz DS. US emergency department visits for outpatient adverse drug events, 2013-2014. JAMA. 2016;316(20):2115-2125.

6. Holbrook A, Schulman S, Witt DM, et al. Evidence-based management of anticoagulant therapy: antithrombotic therapy and prevention of thrombosis, 9th ed: American College of Chest Physicians Evidence-Based Clinical Practice Guidelines. Chest. 2012;141(2 suppl):152S-184S.

7. Pollack CV Jr, Reilly PA, Eikelboom J, et al. Idarucizumab for dabigatran reversal. N Engl J Med. 2015;373(6):511-520.

8. Lu G, DeGuzman FR, Hollenbach SJ, et al. A specific antidote for reversal of anticoagulation by direct and indirect inhibitors of coagulation factor Xa. Nat Med. 2013;19(4):446-451.

9. Yeh CH, Fredenburgh JC, Weitz JI. The real decoy: an antidote for factor Xa-directed anticoagulants. Circ Res. 2013;113(8):954-957.

10. Lu G, DeGuzman FR, Lakhotia S, Hollenbach SJ, Phillips DR, Sinha $\mathrm{U}$. Recombinant antidote for reversal of anticoagulation by factor Xa inhibitors. Blood. 2008;112(11):983.

11. Lu G, DeGuzman FR, Hollenbach SJ, et al. Reversal of low molecular weight heparin and fondaparinux by a recombinant antidote (r-Antidote, PRT064445). Circulation. 2010;122(suppl 21):A12420.

12. Lu G, Luan P, Hollenbach S, et al. Reconstructed recombinant factor $\mathrm{Xa}$ as an antidote to reverse anticoagulation by factor Xa inhibitors. J Thromb Haemost. 2009; 7:309.
13. Hollenbach S, Lu G, Deguzman F, Curnutte J, Conley P, Sinha U. Bolus administration of PRT064445, a recombinant factor Xa inhibitor antidote, reverses blood loss and PD markers in a rat model following enoxaparin-induced anticoagulation. Eur Heart J. 2012;33:309-310.

14. Hollenbach SJ, Lu G, Tan S, et al. PRT064445 but not recombinant Fviia reverses rivaroxaban induced anticoagulation as measured by reduction in blood loss in a rabbit liver laceration model. Blood. 2012; 120(21):3414.

15. Lu G, Lin J, Curnutte JT, Conley PB. Reversal of heparin-induced anticoagulation by andexanet alfa, a universal antidote for factor Xa inhibitors. Blood. 2015;126(23):2329.

16. Connolly SJ, Milling TJ Jr, Eikelboom JW, et al. Andexanet alfa for acute major bleeding associated with factor Xa inhibitors. $N$ Engl $J$ Med. 2016;375(12):1131-1141.

17. Siegal DM, Curnutte JT, Connolly SJ, et al. Andexanet alfa for the reversal of factor Xa inhibitor activity. $N$ Engl J Med. 2015;373(25): 2413-2424.

18. Quintard H, Viard D, Drici MD, Ruetsch C, Samama CM, Ichai C. Idarucizumab administration for reversing dabigatran effect in an acute kidney injured patient with bleeding. Thromb Haemost. 2017; 117(1):196-197.

19. Crowther M, Kitt M, Lorenz T, et al. A phase 2 randomized, doubleblind, placebo controlled trial of PRT064445, a novel, universal antidote for direct and indirect factor Xa inhibitors. J Thromb Haemost. 2013;11(suppl 2):30.

20. Crowther M, Kitt M, McClure M, et al. Abstract 10: randomized, doubleblind, placebo-controlled single ascending dose pharmacokinetic and pharmacodynamic study of PRT064445, a universal antidote for factor Xa inhibitors. Arterioscler Thromb Vasc Biol. 2013;33(suppl 1):A10.

21. Crowther M, Levy GG, Lu G, et al. A phase 2 randomized, double-blind, placebo-controlled trial demonstrating reversal of edoxaban-induced anticoagulation in healthy subjects by andexanet alfa (PRT064445), a universal antidote for factor Xa (fXa) inhibitors. Blood. 2014;124(21): 4269.

22. Crowther M, Lu G, Conley P, et al. 455: reversal of factor XA inhibitorsinduced anticoagulation in healthy subjects by andexanet alfa. Crit Care Med. 2014;42(12):A1469.

23. Nafee T, Aslam A, Chi G, et al. Andexanet alfa for the reversal of anticoagulant activity in patients treated with direct and indirect factor Xa inhibitors. Expert Rev Cardiovasc Ther. 2017;15(4):237-245.

24. Smythe MA, Trujillo T, Fanikos J. Reversal agents for use with direct and indirect anticoagulants. Am J Health Syst Pharm. 2016;73(10 suppl 2): S27-S48.

25. Levy JH, Ageno W, Chan NC, Crowther M, Verhamme P, Weitz JI. When and how to use antidotes for the reversal of direct oral anticoagulants: guidance from the SSC of the ISTH. J Thromb Haemost. 2016;14(3):623-627.

26. Milling TJ Jr, Kaatz S. Preclinical and clinical data for factor Xa and "Universal" reversal agents. Am J Med. 2016;129(11s):S80-S88.
Journal of Blood Medicine

Publish your work in this journal

The Journal of Blood Medicine is an international, peer-reviewed, open access, online journal publishing laboratory, experimental and clinical aspects of all aspect pertaining to blood based medicine including but not limited to: Transfusion Medicine; Blood collection, Donor issues, Transmittable diseases, and Blood banking logistics; Immunohematology; Artificial and alternative
Dovepress

blood based therapeutics; Hematology; Biotechnology/nanotechnology of blood related medicine; Legal aspects of blood medicine; Historical perspectives. The manuscript management system is completely online and includes a very quick and fair peer-review system. Visit http://www.dovepress.com/ testimonials.php to read real quotes from published authors. 